УДК 621.314.58:681.5.015

\title{
ГОЛОМОРФНОЕ ПОГРУЖЕНИЕ КАК МЕТОД РАСЧЕТА УСТАНОВИВШИХСЯ РЕЖИМОВ ЭЛЕКТРИЧЕСКИХ СЕТЕЙ НЕФТЯНЫХ И ГАЗОВЫХ МЕСТОРОЖДЕНИЙ И ОЦЕНКИ ИХ УСТОЙЧИВОСТИ
}

\author{
Исаев Юсуп Ниязбекович1, \\ isaev_yusup@mail.ru
}

\author{
Кабалин Дмитрий Андреевич1, \\ mitya.kabalin@gmail.com
}

\author{
Филипас Александр Александрович1, \\ filipas@tpu.ru \\ 1 Национальный исследовательский Томский политехнический университет, \\ Россия, 634050, г. Томск, пр. Ленина, 30.
}

Для эфффективного решения задачи оперативно-диспетчерского управления режимами работы единой энергетической системы, ее отдельных энергосистем и энергорайонов, в частности, энергорайонов нефтедобычи, требуется выполнять расчеты установившихся режимов электрических сетей. Кроме того, наряду с расчетами установившихся режимов важными являются вопросы исследования устойчивости работы энергосети. Сходимость и скорость сходимости широко применяемых итерационных методов расчета установившихся режимов зависят от многих режимных и расчетных фракторов, определяемых параметрами сети и режима, выбором исходных приближений, способом задания исходных данных. Поэтому разработка новых методов, позволяющих рассчитьвать все установившиеся режимы, представляет значительный практический интерес. Одним из перспективных методов является метод голоморфрного погружения. В данном методе неизвестные параметры узлов представляются в виде голоморфных функций, которые можно представить в виде степенных рядов, коэфрфициенты которых рассчитываются по рекуррентным выражениям и задача сводится к нахождению коэфффициентов степенных рядов. В опубликованной ранее статье авторов приведено рассмотрение метода для схемы с нагрузочными узлами. Для полного корректного анализа режимов реальных энергосистем необходимо показать, как нужно вести расчет для генераторных узлов. В работе представлены рекуррентные выражения для расчета неизвестных коэфффициентов голоморфрных фуннкиий неизвестных параметров системы уравнений установившегося режима для нагрузочных и генераторных узлов. Полученные выражения, в отличие от предложенных в работах других авторов, являются более общими. Показан принцип формирования матричного уравнения для нахождения неизвестных коэффрициентов с разделением комплексных параметров на действительную и мнимую части. Предложен способ получения сходящихся степенных рядов искомых функций в отдельных случаях. На примере тестовой энергосистемы показано преимущество перед методом Ньютона-Расрсона. Рассматривается вопрос оиенки существования решения системы уравнений установившегося режима для многоузловой сети на основе сигма-графика. Предложен подход к определению показателя запаса статической устойчивости энергосистемы на основе критерия Фабри.

Цель: применить аналитический метод голоморфного погружения для расчета электрической схемы, содержащей нагрузочные и генераторные узлы; оценить влияние количества рассчитываемых коэффрициентов степенных рядов на точность получаемого решения, а также рассмотреть способы повьшения численной точности решения, рассмотреть вопрос оценки существования решения системы уравнений установившегося режима для многоузловой сети на основе анализа степенных рядов.

Методы: разложение Тейлора, аналитическое продолжение, аппроксимация Паде, решение алгебраических уравнений рекуррентным методом.

Результаты. На примере схемы с плохообусловленной матрицей Якоби, в которой метод Ньютона-Рафсона не сходится с плоского старта, показано преимущество метода голоморфного погружения. Показано влияние количества членов степенных рядов на погрешность расчета. Для рассматриваемой схемы выполнена графрическая оценка существования решения системы уравнений.

Выводы. Для нагрузочных и генераторных узлов неизвестные параметры можно представить в виде голоморфных функций, которые можно записать в виде ряда Тейлора, коэффрициенты которого рассчитываются по рекуррентным выражениям. Частичньй учет шунтов на землю в диагональных элементах матрицы последовательных проводимостей позволяет получить сходящиеся степенные ряды в отдельных случаях. Рассмотренный графический способ оценки возможности существования режима позволяет произвести примерную оценку. В отличие от классических итерационных методов для метода голоморфоного погружения не нужно задавать начальное приближение.

\section{Ключевые слова:}

Энергосистема, установившийся процесс, устойчивость, нелинейные уравнения, голоморфное погружение, разложение Паде, сходимость.

\section{Введение}

Распределительные электрические сети нефтегазовых месторождений выполняются, как правило, радиальными, при этом для них характерно: наличие большого числа ступеней напряжений и ступеней трансформации; большое количество как длинных, так и коротких линий электропередачи разных уровней напряжения, большое количество линий электро- 
передачи, активные составляющие сопротивлений которых близки или превышают по значению реактивные составляющие сопротивлений; высокая неоднородность нагрузки в узлах, заключающаяся в том, что нагрузка в одних узлах может составлять несколько киловатт, в других - десятки мегаватт; сильная нагруженность сети; практическое отсутствие узлов с фиксированным модулем напряжения [1-5]. Отмеченные особенности распределительных электрических сетей нефтегазовых месторождений влияют на матрицы собственных и взаимных проводимостей и матрицу Якоби, существенно ухудшая их обусловленность, и как следствие влияют на сходимость классических итерационных методов расчета установившихся режимов (УР), в частности, на сходимость метода Ньютона-Рафсона, который в настоящее время широко применяется при анализе режимов электроэнергетических систем [1, 2].

Так как для эффективного решения задачи оперативно-диспетчерского управления режимами работы единой энергетической системы в целом, ее отдельных энергосистем и энергорайонов, в частности, энергорайонов нефтедобычи, требуется получение однозначного ответа о возможности существования режима, реализация новых методов, которые позволят рассчитывать все установившиеся режимы, представляет значительный практический интерес. Кроме того, наряду с расчетами УР важными являются вопросы исследования устойчивости работы энергосети, т. к. нарушения устойчивости приводят к торможению и отключению электроприемников объектов нефтегазовой отрасли $[5,6]$. Поэтому работа посвящена расчету УР электрических сетей и вопросам устойчивости функционирования энергосети.

На протяжении нескольких десятилетий ведется разработка новых методов расчета установившихся режимов. Разработаны методы по параметру, а также различные минимизационные методы, основанные на методе Ньютона (метод Левенберга-Марквардта и т. п.) [1-5, 7]. Разработанные методы надежнее по сходимости, чем метод Ньютона-Рафсона. При этом в большинстве случаев их использование позволяет получить решение, когда классический метод НьютонаРафсона расходиться по тем или иным причинам. Однако их использование все же не всегда гарантирует получение однозначного ответа о существовании режима [7]. Кроме того, данные методы в вычислительном плане более затратные. Поэтому вопрос создания более совершенных методов и алгоритмов расчета режимов электрических систем остается актуальным.

В течение последних нескольких лет ведется активная разработка нового метода голоморфного погружения, предложенного испанским исследователем Антонио Триасом $[8,9]$. Данный неитерационный метод основан на теории комплексного анализа и аналитического продолжения. Важной особенностью данного метода является однозначность решения: метод теоретически гарантированно сходится к решению независимо от начального приближения $[8,9]$.
В статье [10] была описана идея метода, рассмотрены вопросы анализа оценки расчетной устойчивости на примере двухузловой схемы, а также приведен пример использования метода голоморфного погружения для трехузловой схемы, содержащей только узлы нагрузки. Поскольку в математических моделях реальных энергосистем кроме нагрузочных узлов имеются генераторные узлы, то для полного корректного анализа режимов реальных энергосистем необходимо их обязательно учитывать, так как они значительно влияют на режим, в частности, при расчете предельных режимов.

\section{Уравнения установившегося режима}

Установившийся режим ЭЭС в общем случае описывается системой нелинейных уравнений узловых напряжений (УУН) [1-5]. Одной из возможных форм записи УУН является форма в виде баланса токов.

Для нерегулируемых узлов (узлы $P Q$-типа), в которых активная и реактивная мощности заданы постоянными величинами, УУН можно записать следующим образом:

$$
\sum_{j=0}^{N-1} Y_{i j} U_{j}=\frac{S_{i}^{*}}{U_{i}^{*}}-Y_{i \sigma} U_{\sigma},
$$

где * - знак сопряжения; $Y_{i j}$ - элементы матрицы узловых проводимостей; $S_{i}=S_{\Gamma i}-S_{\mathrm{H} i}-$ результирующая комплексная мощность, входящая в $i$-й узел; $U_{i}-$ напряжение узла $i ; Y_{i б}$ - взаимная проводимость между узлом $i$ и балансирующим; $U_{6}-$ напряжение балансирующего узла; $N$ - количество узлов в схеме.

Для регулируемых узлов (узлы $P V$-типа), в которых задается активная мощность, модуль напряжения и диапазон регулирования реактивной мощности, систему УУН переписывают следующим образом:

$$
\sum_{j=0}^{N-1} Y_{i j} U_{j}=\frac{P_{i}-j Q_{i}}{U_{i}^{*}}-Y_{i \sigma} U_{\sigma}, i \in P V,
$$

где активная мощность $P_{i}$ является постоянной известной величиной, реактивная мощность $Q_{i}$ является неизвестной переменной, которая определяется в процессе расчета.

Дополнительно для узлов $P V$-типа используют уравнения, связывающие комплекс напряжения в узле с его модулем:

$$
\left|U_{i}\right|^{2}=\left|U_{\text {зд } i}\right|^{2}=U_{i} U_{i}^{*}, i \in P V .
$$

Модуль и фазовый угол напряжения балансирующего (базисного) узла задается, при этом фазовый угол обычно принимается равным нулю:

$$
U_{\text {б }}=U_{\text {зді }}, i \in \text { б.у. }
$$

Задача расчета УР сводится к решению системы уравнений (1)-(4), решением которой являются комплексы напряжений в узлах сети. На основе полученных напряжений рассчитываются остальные параметры режима электрической сети - перетоки и токи в ветвях и т. д.

Критерием получения решения является условие соблюдения небалансов мощности в узлах сети: 


$$
\max \left(\left|P_{i}-P_{i \text { pacu }}\right| ;\left|Q_{i}-Q_{i \text { pacu }}\right|\right) \leq \varepsilon,
$$

где $P_{i}$ и $Q_{i}$ - узловая активная и реактивная мощность $i$-го узла соответственно; $P_{i \text { расч }}$ и $Q_{i \text { расч }}-$ расчетная узловая активная и реактивная мощность $i$-го узла соответственно; $\varepsilon$ - заданная допустимая погрешность по мощности, допустимое значение $\varepsilon=10^{-4} \ldots 10^{-3}$.

Голоморфное погружение уравнений установившегося режима в комплексную плоскость

Рассмотрим применение метода голоморфного погружения для решения системы УУН в форме баланса токов. Осуществляем голоморфное погружение уравнений в комплексную плоскость. Голоморфное погружение заключается во введении в исходную систему алгебраических уравнений комплексного параметра $\alpha$ таким образом, что [8]:

- напряжения и реактивная мощность генераторных узлов представляются в виде функций от этого комплексного параметра $\alpha$, при этом данные функции $f(\alpha)$ должны быть голоморфны. Кроме того, комплексно-сопряженные функции напряжений также должны быть голоморфными. Для этого эти функции должны удовлетворять условию Коши-Римана [9]

$$
\begin{gathered}
\frac{\partial f(\alpha)}{\partial \alpha^{*}}=0 \rightarrow\left\{\begin{array}{l}
\frac{\partial u}{\partial x}=\frac{\partial v}{\partial y} \\
\frac{\partial u}{\partial y}=-\frac{\partial v}{\partial x}
\end{array}\right. \\
f(\alpha)=u(x, y)+j v(x, y), \alpha=x+j y .
\end{gathered}
$$

Голоморфные функции определены на открытом подмножестве комплексной плоскости $\alpha$ и комплексно дифференцируемы в каждой точке этого подмножества. Голоморфные функции являются аналитическими и могут быть представлены в виде сходящегося в некоторой окрестности каждой точки ряда Тейлора [9]. Данное свойство голоморфных функций очень важно в предлагаемом методе.

- при подстановке параметра погружения $\alpha=0$ в «погруженную» систему уравнений данная нелинейная система должна преобразовываться в линейную, математически точное решение которой может быть определено. При $\alpha=0$ система соответствует сети без нагрузки и генерации (все инъекции узловых мощностей исчезают). При $\alpha=1$ система уравнений соответствует исходной системе алгебраических уравнений.

В общем голоморфное погружение комплексного параметра $\alpha$ в исходную систему алгебраических уравнений может быть выполнено любым способ, главное, чтобы выполнялись описанные требования [8]

Следует отметить, что поскольку задача в дальнейшем сводится к работе со степенными рядами, то для получения сходящихся рядов, а также чтобы первые коэффициенты функций напряжений были равны (или близки) единице в ситуации без нагрузки и генерации при $\alpha=0$, необходимо разделить матрицу проводимости $Y$ на матрицу последовательных проводи- мостей $Y_{\text {п }}$ и матрицу шунтов $Y_{\text {ш: }} Y=Y_{\text {п }}+\operatorname{diag}\left(Y_{\text {ш }}\right)$ [10]. Тогда правую часть в (1) и (2) можно представить в следующем виде:

$$
\sum_{j=0}^{N-1} Y_{i j} U_{j}=\sum_{j=0}^{N-1} Y_{\text {пі }} U_{j}+Y_{\amalg i} U_{i},
$$

где $Y_{\text {шi }}$ - суммарная проводимость на землю узла $i$; $Y_{\text {піj }}$ - элементы матрицы последовательных проводимостей (матрица узловых проводимостей, в диагональных элементах которой не учитываются проводимости на землю).

Применяя описанные правила голоморфного погружения, а также учитывая (6), голоморфное погружение может быть представлено в следующем виде:

$$
\begin{aligned}
& \sum_{j=0}^{N-1} Y_{\mathrm{ni}} U_{j}(\alpha)=\frac{\alpha S_{i}^{*}}{U_{i}^{*}\left(\alpha^{*}\right)}-\alpha Y_{\mathrm{mi}} U_{i}(\alpha)-Y_{i \bar{\sigma}} U_{\bar{\sigma}}(\alpha), \\
& i \in P Q \text {, } \\
& \sum_{j=0}^{N-1} Y_{\mathrm{nij}} U_{j}(\alpha)=\frac{\alpha P_{i}-j Q_{i}(\alpha)}{U_{i}^{*}\left(\alpha^{*}\right)}-\alpha Y_{\mathrm{m} i} U_{i}(\alpha)-Y_{i \bar{\sigma}} U_{\bar{\sigma}}(\alpha), \\
& i \in P V \text {, } \\
& U_{i}(\alpha) U_{i}^{*}\left(\alpha^{*}\right)=\left|U_{i 0}\right|^{2}+\alpha\left(\left|U_{\text {зді }}\right|^{2}-\left|U_{i 0}\right|^{2}\right), i \in P V,(9) \\
& U_{6}(\alpha)=1+\alpha\left(U_{6}-1\right),
\end{aligned}
$$

где $\alpha$ - параметр погружения; $U_{\text {зді }}$ - заданное напряжение узла, $P_{i}=P_{г і}-P_{\text {ні и }} Q_{i}=Q_{\text {гі }}-Q_{\text {ні }}-$ активная и реактивная мощность, входящая в $i$-й узел; $U_{i}(\alpha)$ и $Q_{i}(\alpha)-$ голоморфные функции напряжения и реактивной мощности от параметра $\alpha ; U_{i}^{*}\left(\alpha^{*}\right)$ - голоморфная комплексно-сопряженная функция напряжения, $U_{i 0}-$ расчетное напряжение $i$-го генераторного узла при подстановке в (7) и (8) параметра погружения $\alpha=0$. Голоморфные функции могут быть представлены в виде сходящегося в некоторой окрестности каждой точки ряда Маклорена, который является частной формой ряда Тейлора [9]:

$$
\begin{gathered}
U_{i}(\alpha)=\sum_{i=0}^{n} U_{i}[n] \alpha^{n}=U_{i}[0]+U_{i}[1] \alpha+\ldots+U_{i}[n] \alpha^{n}, \\
i \in P Q+P V+\sigma . y . \\
Q_{i}(\alpha)=\sum_{i=0}^{n} Q_{i}[n] \alpha^{n}=Q_{i}[0]+Q_{i}[1] \alpha+\ldots+Q_{i}[n] \alpha^{n}, \\
i \in P V .
\end{gathered}
$$

Следует заметить, что в формулах (7) и (8) комплексно-сопряженная функция напряжения представлена в виде $U_{i}^{*}\left(\alpha^{*}\right)=\sum_{i=0}^{n} U_{i}^{*}[n] \alpha^{n}$, а не в виде $U_{i}^{*}(\alpha)=\sum_{i=0}^{n} U_{i}^{*}[n]\left(\alpha^{*}\right)^{n}$, так как именно функция $U_{i}^{*}\left(\alpha^{*}\right)$ удовлетворяет условию Коши-Римана (5).

Введем вспомогательную функцию $W_{i}^{*}\left(\alpha^{*}\right)$, обратную $U_{i}^{*}\left(\alpha^{*}\right)$ которую также можно разложить в степенной ряд: 


$$
W_{i}^{*}\left(\alpha^{*}\right)=\frac{1}{U_{i}^{*}\left(\alpha^{*}\right)}=W_{i}^{*}[0]+W_{i}^{*}[1] \alpha+\ldots+W_{i}^{*}[n] \alpha^{n} .
$$

Неизвестные коэффициенты функции $W_{i}^{*}\left(\alpha^{*}\right)$ можно найти из равенства $W_{i}^{*}\left(\alpha^{*}\right) \cdot U_{i}^{*}\left(\alpha^{*}\right)=1$. Далее знак сопряжения опускаем. Расписав функции в виде рядов, открыв скобки и приравняв коэффициенты при одинаковых степенях параметра $\alpha$ с обеих сторон уравнения, получаем общее выражение для вычисления $n$-го коэффициента ряда функции $W(\alpha)[11-16]$ :

$$
W[n]=\left\{\begin{array}{l}
\frac{1}{U[0]}, n=0 ; \\
-\frac{\sum_{k=0}^{n-1} W[k] U[n-k]}{U[0]}, n \geq 1 .
\end{array}\right.
$$
нения

Подставляем (10)-(13) в (7) и (8), получаем урав-

$$
\begin{aligned}
& \sum_{j=0}^{N-1} Y_{\mathrm{mij}}\left(U_{j}[0]+U_{j}[1] \alpha+\ldots+U_{j}[n] \alpha^{n}\right)= \\
& =\alpha S_{i}^{*}\left(W^{*}[0]+W^{*}[1] \alpha+\ldots+W^{*}[n] \alpha^{n}\right)- \\
& -\alpha Y_{\mathrm{mi}}\left(U_{i}[0]+U_{i}[1] \alpha+\ldots+U_{i}[n] \alpha^{n}\right)- \\
& \quad-Y_{i \bar{\sigma}}\left(1+\alpha\left(U_{\sigma}-1\right)\right), i \in P Q, \\
& \sum_{j=0}^{N-1} Y_{\mathrm{n} i j}\left(U_{j}[0]+U_{j}[1] \alpha+\ldots+U_{j}[n] \alpha^{n}\right)= \\
& =\left(\alpha P_{i}-j\left(Q[0]+Q[1] \alpha+\ldots+Q[n] \alpha^{n}\right) \times\right. \\
& \quad \times\left(W_{i}^{*}[0]+W_{i}^{*}[1] \alpha+\ldots+W_{i}^{*}[n] \alpha^{n}\right)- \\
& -\alpha Y_{\text {шi }}\left(U_{i}[0]+U_{i}[1] \alpha+\ldots+U_{i}[n] \alpha^{n}\right)- \\
& \quad-Y_{i \bar{\sigma}}\left(1+\alpha\left(U_{\sigma}-1\right)\right) i \in P V .
\end{aligned}
$$

Раскрывая скобки в (14) и (15) и приравнивая коэффициенты при одинаковых степенях $a^{n}$, получаем: - для узлов $P Q$-типа:

$$
\begin{gathered}
\sum_{j=0}^{N-1} Y_{\mathrm{mi} j} U_{j}[n]= \\
=\left\{\begin{array}{l}
-Y_{i \bar{\sigma}}, n=0 ; \\
S_{i}^{*} W^{*}[n-1]-Y_{\mathrm{mi}} U_{i}[n-1]^{n}-Y_{i \bar{\sigma}}\left(U_{\sigma}-1\right), n=1 ; \\
S_{i}^{*} W_{i}^{*}[n-1]-Y_{\mathrm{mi}} U_{i}[n-1], n>1 ;
\end{array}\right.
\end{gathered}
$$

- для узлов $P V$-типа:

$$
\begin{gathered}
\sum_{j=0}^{N-1} Y_{\mathrm{nij}} U_{j}[n]= \\
=\left\{\begin{array}{c}
P_{i}[0] W_{i}^{*}[0]-Y_{i \bar{\sigma}}, n=0 ; \\
P_{i} W_{i}^{*}[0]-j\left(Q[0] W_{i}^{*}[1]+Q[1] W_{i}^{*}[0]\right)- \\
-Y_{m i} U_{i}[0]-Y_{i \bar{\sigma}}\left(U_{\sigma}-1\right), n=1 ; \\
P_{i} W_{i}^{*}[n-1]- \\
-j \sum_{k=0}^{n} Q_{i}[k] W_{i}^{*}[n-k]-Y_{\mathrm{m} i} U_{i}[n-1], n>1 .
\end{array}\right.
\end{gathered}
$$

Из уравнений (16) и (17) видно, что исходная задача сводится к нахождению коэффициентов степенных рядов. При этом мы можем точно определить нулевые коэффициенты степенных рядов напряжений и неизвестных реактивных мощностей. Уравнения (16) и (17) при $n=0$ соответствуют подстановке в «погруженную» систему уравнений параметра $\alpha=0$. Нулевые коэффициенты реактивных мощностей $Q_{j}[0]=0, j \in P V$, так как при $\alpha=0$ инъекции мощности генерации отсутствуют. Нулевые коэффициенты степенных рядов напряжений определяются путем решения уравнения

$$
\sum_{j=0}^{N-1} Y_{\text {пі }} U_{j}[0]=-Y_{i \sigma}, \quad j \in P Q+P V .
$$

В случае если в матрице последовательных проводимостей диагональные элементы не содержат шунтов на землю (даже шунтов трансформаторных ветвей П-образной схемы замещения) и равны сумме проводимостей ветвей, связанных с соответствующим узлом, с обратным знаком, тогда нулевые коэффициенты степенных рядов напряжений равны единице: $U_{i}[0]=1, j \in P Q+P V+б$. . Если же в диагональных элементах матрицы последовательных проводимостей частично учесть шунты на землю (с целью получения сходящихся степенных рядов в отдельных случаях), то нулевые коэффициенты степенных рядов напряжений $U_{i}[0] \neq 1$, а являются комплексными числами, действительная часть которых близка к 1 . В отличие от [12-16], где предлагается полностью выделять шунты на землю из диагональных элементов матрицы узловых проводимостей и принимать $U_{i}[0]=1$, предложенный способ частичного учета шунтов на землю и расчета нулевых коэффициентов степенных рядов неизвестных позволяет получить сходящиеся степенные ряды в отдельных случаях, тем самым получить корректный результат.

Зная нулевые коэффициенты степенных рядов, можно рассчитать остальные коэффициенты. Для узлов $P Q$-типа из (16) видно, что неизвестные $n$-ые коэффициенты функций напряжений $U_{i}(\alpha)$ можно вычислить через известные $(n-1)$-е коэффициенты по рекуррентному выражению. Для узлов $P V$-типа из (17) видно, что при вычислении коэффициента $n$-го порядка степенного ряда напряжения $U_{j}[n]$ требуются коэффициенты $n$-го порядка $W_{i}^{*}[n]$ и $Q_{i}[n]$. Так как коэффициенты $Q_{j}[n]$ являются искомыми, то перенесем их в правую часть выражения (17), учитывая $Q_{i}[0]=0$, получаем

$$
\begin{gathered}
\sum_{j=0}^{N-1} Y_{\text {піj }} U_{j}[n]+j Q_{i}[n] W_{i}^{*}[0]= \\
+\left\{\begin{array}{l}
-Y_{i \bar{\sigma}}, n=0 ; \\
P_{i} W_{i}^{*}[0]-Y_{\text {шi }} U_{i}[0]-Y_{i \sigma}\left(U_{\sigma}-1\right), n=1 ; \\
P_{i} W_{i}^{*}[n-1]- \\
-j \sum_{k=1}^{n-1} Q_{i}[k] W_{i}^{*}[n-k]-Y_{\amalg i} U_{i}[n-1], n>1 .
\end{array}\right.
\end{gathered}
$$


В уравнении (18) нам удалось избавиться от неизвестного коэффициента $W_{i}^{*}[n]$, который не может быть найден без $U_{i}[n]$. Однако в уравнении (18) у нас две неизвестные переменные $-Q_{i}$ и $U_{i}$. Для того чтобы найти решение, необходимо дополнить систему уравнений уравнением (9). Представим уравнение (9) следующим образом

$$
\begin{aligned}
&\left(U_{i}[0]+U_{i}[1] \alpha+\ldots+U_{i}[n] \alpha^{n}\right) \times \\
& \times\left(U_{i}^{*}[0]+U_{i}^{*}[1] \alpha+\ldots+U_{i}^{*}[n] \alpha^{n}\right)= \\
&=\left|U_{i 0}\right|^{2}+\alpha\left(\left|U_{\text {зді }}\right|^{2}-\left|U_{i 0}\right|^{2}\right), i \in P V .
\end{aligned}
$$

где $U_{i 0}=U_{i}[0]$ - расчетное напряжение $i$-го генераторного узла при $\alpha=0$.

Перемножив скобки в правой части выражения (19) и приравняв коэффициенты при одинаковых степенях параметра $\alpha$, получаем

$$
\begin{gathered}
U_{i}[0] \cdot U_{i}^{*}[n]+U_{i}[n] \cdot U_{i}^{*}[0]= \\
=\left\{\begin{array}{l}
\left|U_{\text {зді }}\right|^{2}-\left|U_{i 0}\right|^{2}, n=1 ; \\
-\sum_{k=1}^{n-1} \dot{U}_{i}[k] U_{i}^{*}[n-k], n>1
\end{array}, \quad i \in P V,\right.
\end{gathered}
$$

Выражения (16), (18) и (20), на основе которых можно найти $n$-е члены степенных рядов функций искомых величин, содержат комплексные переменные, что затрудняет задачу нахождения решения. Выделим действительную и мнимую части в данных уравнениях. Для этого элементы матрицы проводимостей представим в виде $Y_{\text {піij }}=g_{\text {піj }}+j b_{\text {піj }}, n$-е коэффициенты функций $U_{i}(\alpha) \quad$ и $\quad W_{i}(\alpha)-U_{j}[n]=U_{j}{ }^{\prime}[n]+j U_{j}{ }^{\prime \prime}[n]$, $W_{j}[n]=W_{j}^{\prime}[n]+j W_{j}^{\prime \prime}[n]$ соответственно. Введем обозначение, запишем правые части выражений (16), (18) и (20) как $I_{P Q i}[n-1], I_{P V i}[n-1]$ и $U U_{i}^{*}[n-1]$ соответственно,

Для узлов $P Q$-типа систему уравнений (16) можно представить в виде двух уравнений

$$
\begin{aligned}
& \sum_{j=0}^{N-1}\left(g_{\text {пі }{ }_{j}} U_{j}^{\prime}[n]-b_{\text {пі } j} U_{j}^{\prime}[n]\right)=I_{P Q i}^{\prime}[n-1], i \in P Q ; \\
& \left.\sum_{j=0}^{N-1}\left(b_{п і j} U_{j}^{\prime}[n]+g_{\text {піj }} U_{j}^{\prime}[n]\right)=I_{P Q i}^{\prime \prime}[n-1]\right), i \in P Q,
\end{aligned}
$$

где

$$
\begin{gathered}
I_{P Q i}^{\prime}[n-1]=\operatorname{Re}\left(I_{P Q_{i}}[n-1]\right) \\
\text { и } I_{P Q i}^{\prime \prime}[n-1]=\operatorname{Im}\left(I_{P Q_{i}}[n-1]\right)
\end{gathered}
$$

- действительная и мнимая части $I_{P Q i}[n-1]$.

Для узлов $P V$-типа систему уравнений (18) можно представить в виде двух уравнений

$$
\begin{gathered}
\sum_{j=0}^{N-1}\left(g_{\text {пі }} U_{j}^{\prime}[n]-b_{\text {пі }} U_{j}^{\prime \prime}[n]\right)+W_{i}^{\prime}[0] \cdot Q_{i}[n]= \\
=I_{P V i}^{\prime}[n-1], i \in P V ; \\
\sum_{j=0}^{N-1}\left(b_{\mathrm{n} i j} U_{j}^{\prime}[n]+g_{\text {пі }} U_{j}^{\prime \prime}[n]\right)+W_{i}^{\prime}[0] \cdot Q_{i}[n]= \\
=I_{P V i}^{\prime \prime}[n-1], i \in P V,
\end{gathered}
$$

где

$$
\begin{gathered}
I_{P V i}^{\prime}[n-1]=\operatorname{Re}\left(I_{P V i}[n-1]\right) \\
\text { и } I_{P V i}^{\prime \prime}[n-1]=\operatorname{Im}\left(I_{P V i}[n-1]\right)
\end{gathered}
$$

- действительная и мнимая части $I_{P V i}[n-1]$.

Правую часть выражения (20) можно представить через мнимые и действительные части коэффициентов:

$$
\begin{gathered}
U_{i}[0] \cdot U_{i}^{*}[n]+U_{i}[n] \cdot U_{i}^{*}[0]=\left(U_{j}^{\prime}[0]+j U_{j}^{\prime \prime}[0]\right) \times \\
\times\left(U_{j}^{\prime}[n]-j U_{j}^{\prime}[n]\right)+\left(U_{j}^{\prime}[n]+j U_{j}^{\prime \prime}[n]\right) \times \\
\times\left(U_{j}^{\prime}[0]-j U_{j}^{\prime \prime}[0]\right)=2\left(U_{i}^{\prime}[0] U_{i}^{\prime}[n]+U_{i}^{\prime \prime}[0] U_{i}^{\prime \prime}[n]\right) .
\end{gathered}
$$

На основе выражений (21)-(23) можно составить общее линейное матричное уравнение, позволяющее рассчитать $n$-ые члены действительных и мнимых частей функций $U_{i}(\alpha)$ и $Q_{i}(\alpha)$. После перестановки уравнений местами общее линейное матричное уравнение, позволяющее определить $n$-е члены при $n \geq 1$, имеет вид

$$
\begin{aligned}
& {\left[\begin{array}{ll:ll:l}
g_{\text {п } q q} & g_{\text {п } q v} & -b_{\text {п } q q} & -b_{\text {п } q v} & 0 \\
g_{\text {пvq }} & g_{\text {пvv }} & -b_{\text {п } v q} & -b_{\text {пvq }} & W_{v}^{\prime \prime}[0] \\
\hdashline b_{\text {п } q q} & b_{\text {п } q v} & g_{\text {п } q q} & g_{\text {п } q v} & 0 \\
b_{\text {п } v q} & b_{\text {пvv }} & g_{\text {пvq }} & g_{\text {пvv }} & W_{v}^{\prime}[0] \\
\hdashline 0 & U_{v}^{\prime}[0] & 0 & U_{v}^{\prime \prime}[0] & 0
\end{array}\right] \times} \\
& \times\left[\begin{array}{l}
U_{q}^{\prime}[n] \\
U_{v}^{\prime}[n] \\
\hdashline U_{q}^{\prime \prime}[n] \\
U_{v}^{\prime}[n] \\
\hdashline Q_{v}[n]
\end{array}\right]=\left[\begin{array}{l}
I_{P Q q}^{\prime}[n-1] \\
I_{P V v}^{\prime}[n-1] \\
\hdashline I_{P Q q}^{\prime \prime}[n-1] \\
I_{P V_{v}}^{\prime \prime}[n-1] \\
\hdashline \frac{1}{2} U U_{v}^{*}[n-1]
\end{array}\right]
\end{aligned}
$$

Общее количество уравнений в линейной системе (24) равно $2 n_{P Q}+3 n_{P V}$, где $n_{P Q}-$ количество узлов $P Q$-типа в схеме, $n_{P V}-$ количество узлов $P V$-типа в схеме. В системе (24) индекс $q \in P Q$, а индекс $v \in P V$. Для решения представленной системы линейных алгебраических уравнений необходимо использовать $L U$-разложение. Стоит отметить, что матрица коэффициентов в системе (24) является неизменной и ее разложение выполняется только один раз, в отличие от метода Ньютона, где факторизация матрицы Якоби осуществляется на каждой итерации расчета.

\section{Аналитическое продолжение и аппроксимация Паде}

Значительную роль в методе голоморфного погружения занимает аналитическое продолжение степенного ряда. В общем случае после вычисления достаточного количества членов степенного ряда, для того чтобы найти решение УУР, необходимо рассчитать значения полученных функций при $\alpha=1$. Подставляя в (11) и (12) значение параметра, получаем, что расчетные значения искомых величин определяются как сумма рассчитанных коэффициентов: $U_{i}=\sum_{i=0}^{n} U_{i}[n]$ и $Q_{i}=\sum_{i=0}^{n} Q_{i}[n]$. Это возможно, если 
радиус сходимости степенных рядов будет больше 1. Если данное условие не будет выполняться, то, для того чтобы определить значение функции за пределами радиуса сходимости, применяется аналитическое продолжение. Аналитическое продолжение - это процесс, посредством которого голоморфная функция, определённая в некоторой области пространства, расширяется за пределы этой области (рис. 1). В качестве аналитического продолжения используется аппроксимация Паде, т. к. из теории сходимости Шталя диагональные аппроксимация Паде дают максимальное аналитическое продолжение [8, 17-21].

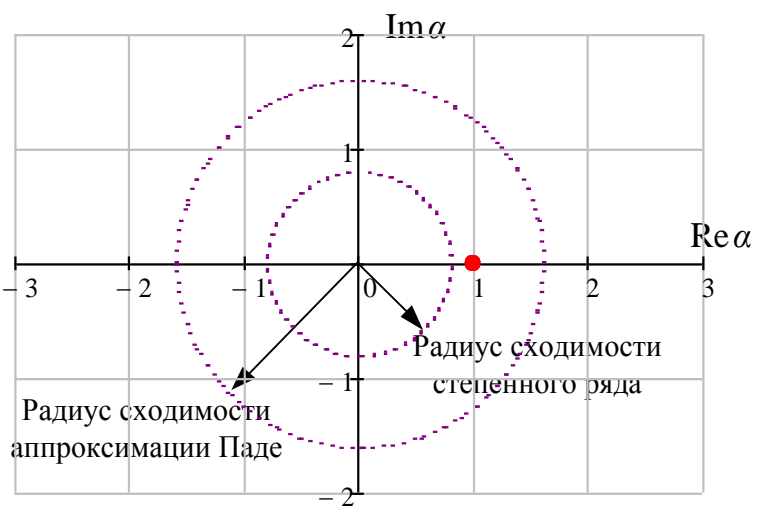

Pис. 1. Радиус сходимости исходного степенного ряда и соответствующей аппроксимации Паде

Fig. 1. Convergence radius of the original power series and the corresponding Padé approximants

Аппроксимация Паде представляет собой функцию в виде отношения двух полиномов [17-19]:

$$
\begin{gathered}
U(\alpha)=\sum_{n=0}^{L+M} U[n] \alpha^{n}=[L / M]_{U(\alpha)}=\frac{a(\alpha)}{b(\alpha)}= \\
=\frac{a[0]+a[1] \alpha+a[2] \alpha^{2}+\ldots+a[L] \alpha^{L}}{b[0]+b[1] \alpha+b[2] \alpha^{2}+\ldots+b[M] \alpha^{M}},
\end{gathered}
$$

где $n$ - степень исходного степенного ряда; $L$ и $M-$ порядок степени полинома числителя и знаменателя соответственно, при этом $n=L+M$. По условию теоремы Шталя [20, 21], наилучшая сходимость обеспечивается, если степени полиномов числителя и знаменателя одинаковы, то есть $L=M$. Такой вид разложения Паде называется диагональным. Основное преимущество аппроксимации Паде состоит в том, что она обеспечивает хорошее приближение вне радиуса сходимости степенного ряда, а также ускоряет сходимость.

Коэффициенты полиномов числителя и знаменателя аппроксимации Паде определяются через найденные ранее коэффициенты степенных рядов функций. Существуют разные способы вычисления аппроксимации Паде. Одним из наиболее простых является матричный способ [17].

Умножая обе стороны (25) на $b(\alpha)$, получаем соотношение

$$
\begin{gathered}
\left(b[0]+b[1] \alpha+\ldots+b[M] \alpha^{M}\right) \times \\
\times\left(U[0]+U[1] \alpha+\ldots+U[L+M] \alpha^{L+M}\right)= \\
=a[0]+a[1] \alpha+\ldots+a[L] \alpha^{L} .
\end{gathered}
$$

Приравнивая коэффициенты при одинаковых степенях $\alpha^{n}$, получаем систему уравнений:

$$
\begin{array}{l|ccc}
1 & a[0] & = & b[0] U[0] \\
\alpha & a[1] & = & b[1] \cdot U[0]+b[0] \cdot U[1] \\
\vdots & \vdots & \vdots & \vdots \\
\alpha^{L} & a[L]= & b[L] U[0]+b[L-1] \times \\
& & & \times U[1]+\cdots+b[0] U[L] \\
\alpha^{L+1} & 0 & = & b[M] U[L-M+1]+b[M-1] \times \\
& & \times U[L-M+2]+\ldots+b[0] U[L+1] \\
\alpha^{L+2} & 0 & = & b[M] U[L-M+2]+b[M-1] \times \\
& & \times U[L-M+3]+\ldots+b[0] U[L+2] \\
\vdots & \vdots & \vdots \\
\alpha^{L+M} & = & & b[M] U[L]+b[M-1] \times \\
& & & \times U[L+1]+\ldots+b[0] U[L+M] .
\end{array}
$$

Система (26) состоит из $L+M+1$ уравнений и имеет $L+M+2$ неизвестных $(a[0], a[1], \ldots, a[L], b[0], b[1], \ldots, b[L])$. Поэтому принимается $b[0]=1$. Из уравнений при $\alpha^{L+1}, \alpha^{L+2}, \ldots \alpha^{L+M}$ можно определить неизвестные коэффициенты полинома знаменателя $b[1], b[2], \ldots, b[M]$. Для этого решается система $M$ линейных уравнений с $M$ неизвестными коэффициентами знаменателя

$$
\begin{gathered}
\left(\begin{array}{cccc}
U[L-M+1] & U[L-M+2] & \cdots & U[L] \\
U[L-M+2] & U[L-M+3] & \vdots & U[L+1] \\
\vdots & \vdots & \ddots & \vdots \\
U[L] & U[L+1] & \cdots & U[L+M-1]
\end{array}\right) \times \\
\times\left(\begin{array}{c}
b[M] \\
b[M-1] \\
\vdots \\
b[1]
\end{array}\right)=-\left(\begin{array}{c}
U[L+1] \\
U[L+2] \\
\vdots \\
U[L+M]
\end{array}\right) .
\end{gathered}
$$

или

$$
\mathbf{A} \cdot \mathbf{X}=\mathbf{B} \text {, }
$$

где $U[i]-$ комплексные коэффициенты степенного ряда, $i=0,1,2, \ldots, L+M$.

В (27) матрица коэффициентов, в зависимости от параметров режима, может быть плохо обусловленной, поэтому задачу расчета неизвестных коэффициентов полинома знаменателя можно назвать некорректной задачей, а решаемое в данном случае уравнение - некорректным алгебраическим уравнением. Для подавления ошибки, появляющейся при вычислении вектора коэффициентов $\mathbf{X}$, за счет проявления ложной высокочастотной составляющей, необходимо осуществить слабый спектральный сдвиг в выражении (27)

$$
\mathbf{X}=\left(\mathbf{A}^{*} \mathbf{A}+\lambda \mathbf{E}\right)^{-1} \mathbf{A}^{*} \mathbf{B},
$$

где $\mathbf{E}$ - единичная матрица; $\lambda$ - параметр регуляризации. В нашем случае параметр регуляризации был выбран $\lambda=10^{-25} \ldots 10^{-10}$ [22]. 
Определив коэффициенты полинома знаменателя, можно найти коэффициенты полинома числителя $a[0]$ $a[1], a[2], \ldots, a[L]$ сравнением коэффициентов при 1 , $\alpha, \alpha^{2}, \ldots, \alpha^{L}$. Формула для определения коэффициентов полинома числителя:

$$
a[L]=\sum_{k=0}^{L} b[k] \cdot U[L-k] .
$$

Таким образом, определив коэффициенты полиномов числителя и знаменателя аппроксимации Паде, путем подстановки $\alpha=1$ в (25), мы можем вычислить искомое значение напряжения в $i$-м узле:

$$
U_{i}=\sum_{n=0}^{L} a_{i}[n] / \sum_{n=0}^{L} b_{i}[n] .
$$

\section{Применение метода голоморфного погружения на тестовых схемах}

Для проверки метода использовались 14-, 30-, 118-, 145-, 300-узловые тестовые схемы IEEE. При этом результаты расчета УР сравнивались с результатами, получаемыми методом Ньютона-Рафсона. Для всех этих схем результаты расчета УР методом голоморфного погружения совпал с результатами, полученными методом Ньютона-Рафсона.

Чтобы показать преимущество рассматриваемого метода голоморфного погружения, рассмотрим схему электрической сети, состоящую из 22 узлов и 22 ветвей и представленную на рис. 2. В рассматриваемой схеме один узел $P V$-типа, двадцать узлов $P Q$-типа, базисным (балансирующим) является узел 1. На рис. 2: над ветвями линий приведено сопротивление $Z_{\text {л }}=R+j X$, под - проводимость на землю $B_{c}$ (положительное значение $B_{c}$ соответствует емкости); над трансформаторными ветвями приведено сопротивление $Z_{\text {тр }}=R+j X$, под - коэффициент трансформации $K_{\text {тр }}$. Bce параметры приведены в о.е. Мощности нагрузки и генерации приведены к базисной величине $S_{\text {баз }}=100$ MВ А. Номинальное напряжение узлов 1-4, 20 составляет 220 кВ; узлов 5-18, 21 - 110 кВ; узла 22 - 35 кВ; узла $19-6$ кВ.

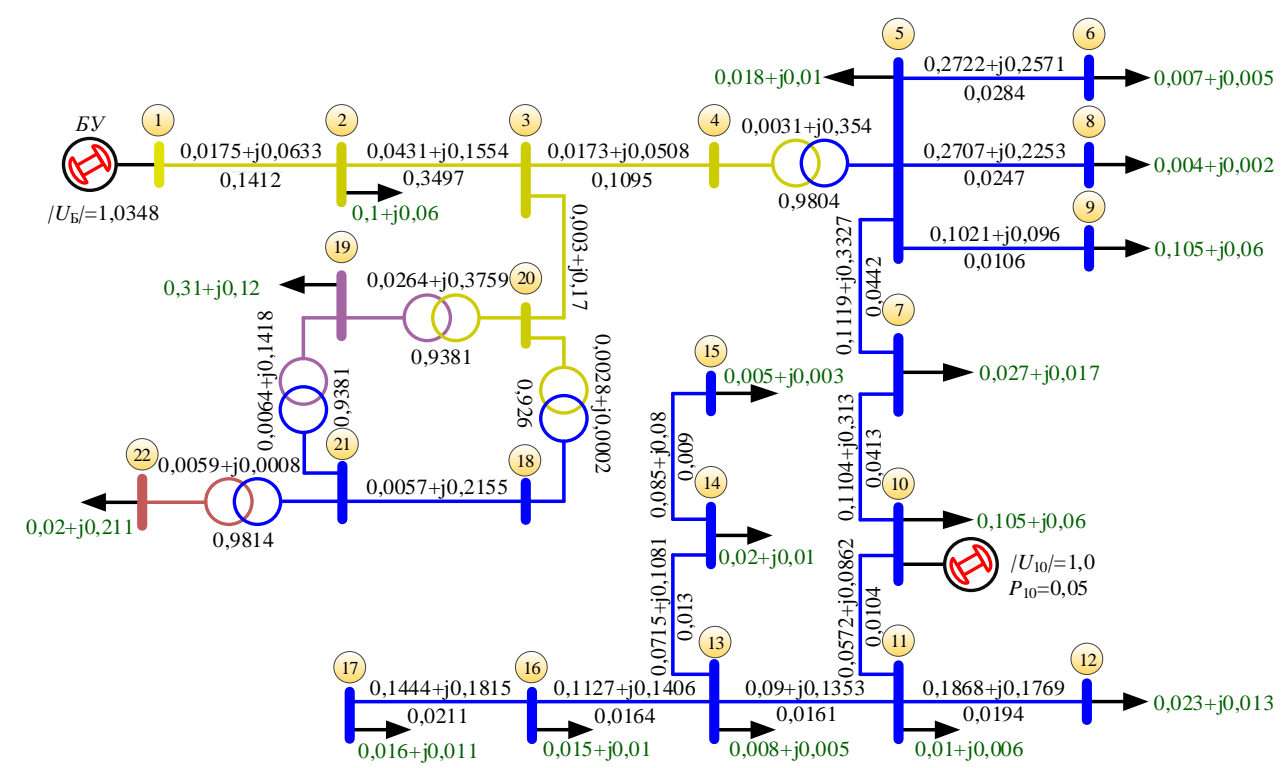

Pис 2. Тестовая схема электрической сети

Fig. 2. Test circuit of the electrical power system

При выполнении расчета УР методом НьютонаРафсона с плоского старта $\left(U_{i}=U_{\text {ном }}\right)$, итерационный процесс не сходится. При этом решение имеет колебательный характер. При этом якобиан на отдельных итерациях меняет знак, что говорит о том, что процесс уже не сойдется к решению [3]. Для данной схемы на сходимость итерационного процесса огромное влияние оказало неудачное начальное приближение. Выполнив подбор начального приближения или применив «стартовый алгоритм» [1], удается получить устойчивое решение.

Расчет УР методом голоморфного погружения выполним двумя способами: первый способ - в векторе шунтов $Y_{\text {ш }}$ не будем учитывать шунты на землю схем замещения трансформаторных ветвей, второй способ - в векторе шунтов $Y_{\text {шI }}$ учитываются все суммар- ные шунты на землю. При расчете получилось, что итоговые значения напряжений в узлах сети совпадают и соответствуют значениям, полученным методом Ньютона-Рафсона с подобранным начальным приближением. Однако результаты расчета коэффициентов степенных рядов отличаются: в первом случае получаются сходящиеся ряды, во втором - расходящиеся. Несмотря на то, что во втором случае ряды расходящиеся, применение аппроксимации Паде позволило получить решение, но при этом погрешность по мощности в узлах больше. На рис. 3 представлен график степенного ряда напряжения и соответствующая ему аппроксимация Паде для узла 19. Отметим, что в первом случае аналитическое продолжение путем рациональной аппроксимации Паде не требуется, поскольку все степенные ряды уже сходятся при $\alpha=1$. 
Однако аппроксимации Паде намного эффективнее оценивают эти функции, поскольку они сходятся к решению с гораздо большей скоростью, чем исходный степенной ряд.

При расчете вторым способом получен правильный конечный результат, с точки зрения математики, однако полученные степенные ряды функций напряжений не имеют физического смысла, в отличие от степенных рядов функций напряжений, полученных при расчете первым способом. Коэффициент погружения $\alpha$ можно рассматривать как коэффициент масштабирования нагрузки и генерации. При увеличении $\alpha$ получается, что во всей схеме происходит пропорциональное утяжеление режима, при этом напряжение должно снижаться, что мы и наблюдаем на рис. 3, $а$. При этом значение параметра $\alpha$, при котором происходит резкое изменение и в дальнейшем резкий скачок графика аппроксимации Паде, можно рассматривать как предельное, соответствующее случаю нарушения устойчивости нагрузки в узле.
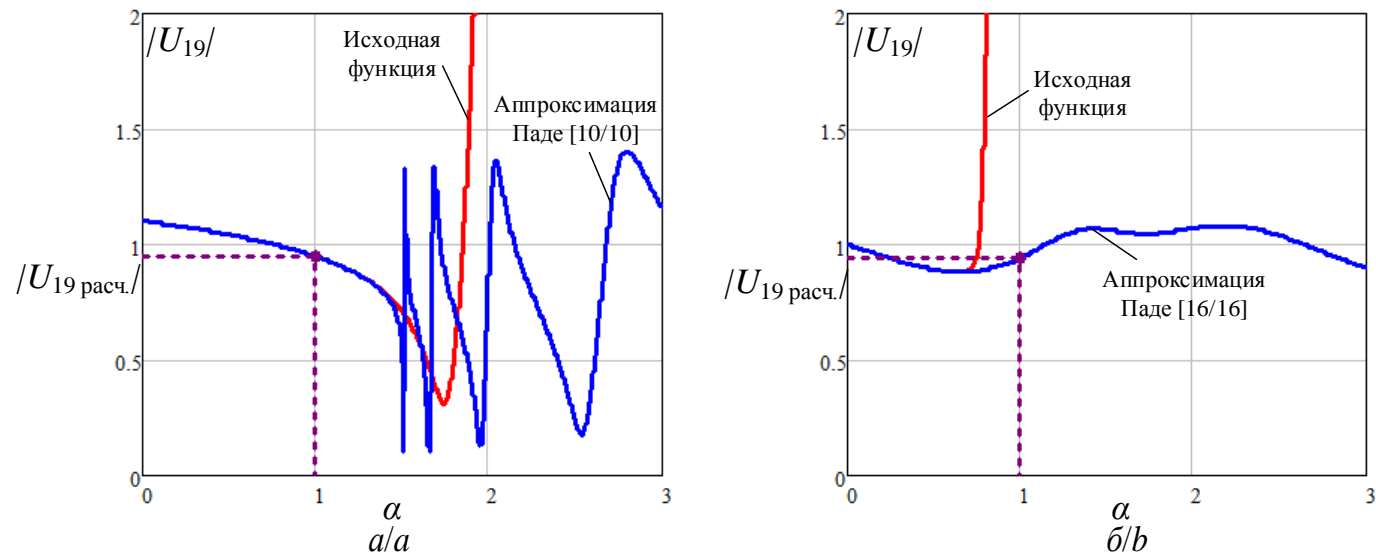

Pис 3. График степенного ряда напряжения и соответствующая ему аппроксимация Паде для узла 19: а) без учета

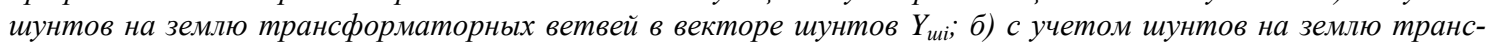
форматорных ветвей в векторе шунтов $Y_{\text {ші }}$

Fig. 3. Picture of power series of the voltage and the corresponding Pade approximation for node 19: a) without shunts to earth of transformer branches in the shunt $\left.Y_{u i} ; b\right)$ with shunts to earth of transformer branches in the shunt $Y_{u i}$

Следует отметить также влияние количества коэффициентов степенных рядов на точность получаемого решения, оцененную по погрешности мощности в узлах. В первом случае минимальное значение максимального небаланса мощности в узлах составляет $5,62 \cdot 10^{-8}$ при $n=20$, во втором $-5,707 \cdot 10^{-3}$ при $n=32$. При этом поначалу с увеличением количества коэффициентов степенного ряда точность повышается, однако затем она начинает снижаться (рис. 4). Это связано с влиянием числовой точности на результаты вычисления аппроксимаций Паде, вычисление которых требует большой точности. Максимальное количество членов степенного ряда ограничивается точностью числа с плавающей запятой. Дальнейший расчет дополнитель- ных членов может приводить к искажению результатов. Для получения более точного решения можно использовать числа повышенной точности или применить метод регуляризации решения. На рис. 4 приведены графики изменения наибольшего небаланса мощности в узлах от количества членов степенного ряда без регуляризации и с регуляризацией при различных параметрах регуляризации $\lambda$. Достаточное количество вычисляемых членов степенного ряда для обеспечения допустимой погрешности по мощности в узлах в общем случае зависит от схемы и режимной ситуации. Примерное количество членов ряда, при котором обеспечивается допустимая погрешность, 20-30. Оптимальное значение параметра регуляризации $\lambda=10^{-15}$.
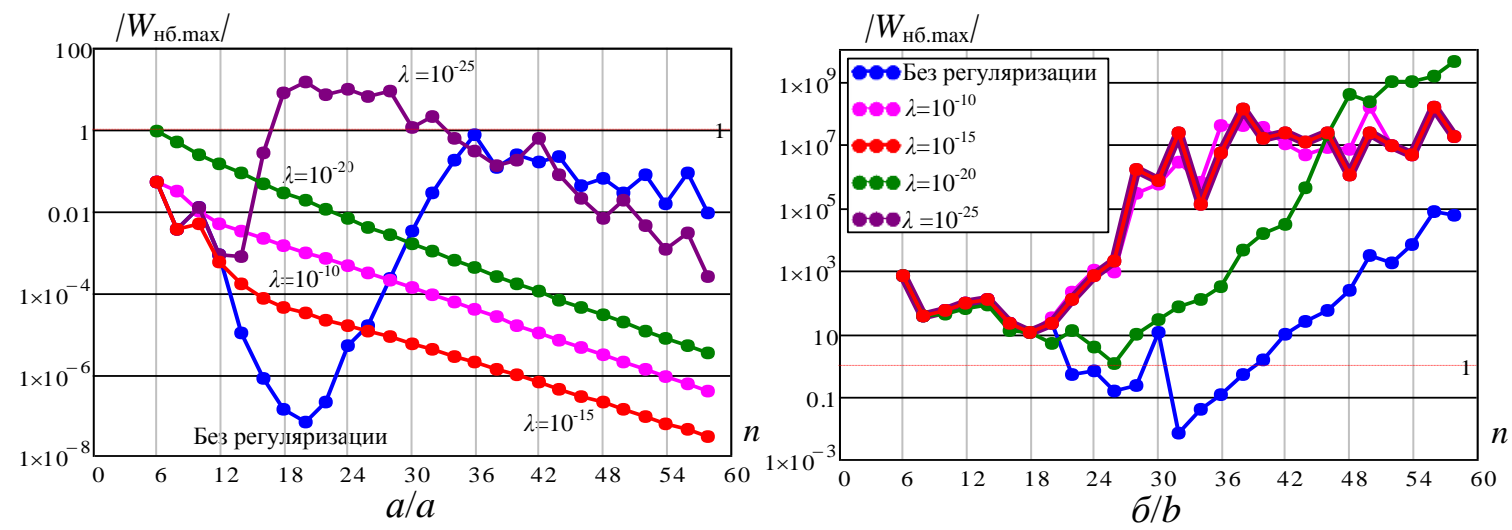
Рис 4. График изменения наибольшего небаланса мощности в узлах от количества членов степенного ряда и пара-

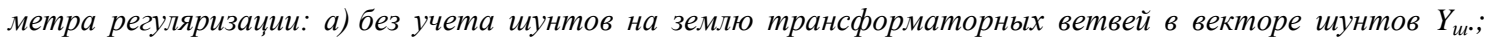
б) с учетом шунтов на землю трансформаторных ветвей в векторе шунтов $Y_{\text {ші }}$

Fig. 4. Picture of the largest power mismatch in nodes as a function of the number of power series members: a) without shunts to earth of transformer branches in the shunt $Y_{u i} ; b$ ) with shunts to earth of transformer branches in the shunt $Y_{u i}$

\section{Оценка существования системы уравнений многоузловой схемы}

Среди проблем, возникающих при анализе режимов, особое место занимает проблема существования решения уравнений УР ЭЭС $[2,3]$. Система уравнений УР может иметь множество решений или не иметь ни одного. Вопрос о существовании нормального режима сводится к определению условий, при которых существует решение этой системы, и к выбору из множества возможных решений того, которое соответствует физической сущности решаемой задачи.

В работе [10] было рассмотрено условие существования решения для двухузловой схемы

$$
D=\frac{1}{4}+\sigma_{R}-\sigma_{I}^{2} \geq 0, \text { или } \sigma_{R} \leq \sigma_{I}^{2}-\frac{1}{4},
$$

где $\sigma_{R}=\operatorname{Re} \sigma=\frac{X Q+R P}{\left|U_{\sigma}\right|^{2}}$ и $\sigma_{I}=\operatorname{Im} \sigma=\frac{X P-R Q}{\left|U_{\sigma}\right|^{2}}-$ действительная и мнимая части коэффициента $\sigma=\frac{Z S^{*}}{\left|U_{\sigma}\right|^{2}}$. Данное условие графически можно представить в виде параболы. Точки внутри параболы соответствуют физическому или устойчивому решению, а точки снаружи - нефизическому решению. Точки, находящиеся на параболе, соответствуют системе на границе устойчивости.

Условие (29) является строгим условием существования режима лишь для двухузловой схемы. Полученное условие можно использовать в качестве практического критерия оценки устойчивости системы уравнений и для многоузловой схемы [23]. Многоузловую схему, состоящую из $n$ узлов, можно преобразовать относительно каждого узла системы к двухузловой схеме (рис. 5) [23-27].

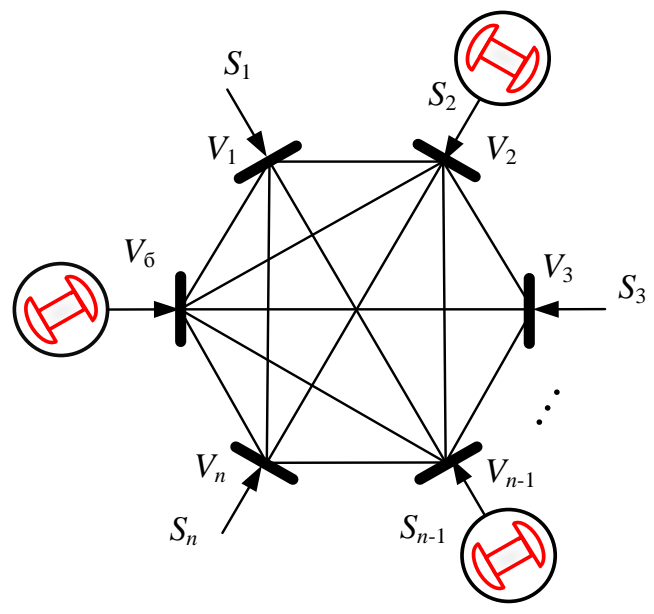

$a / a$
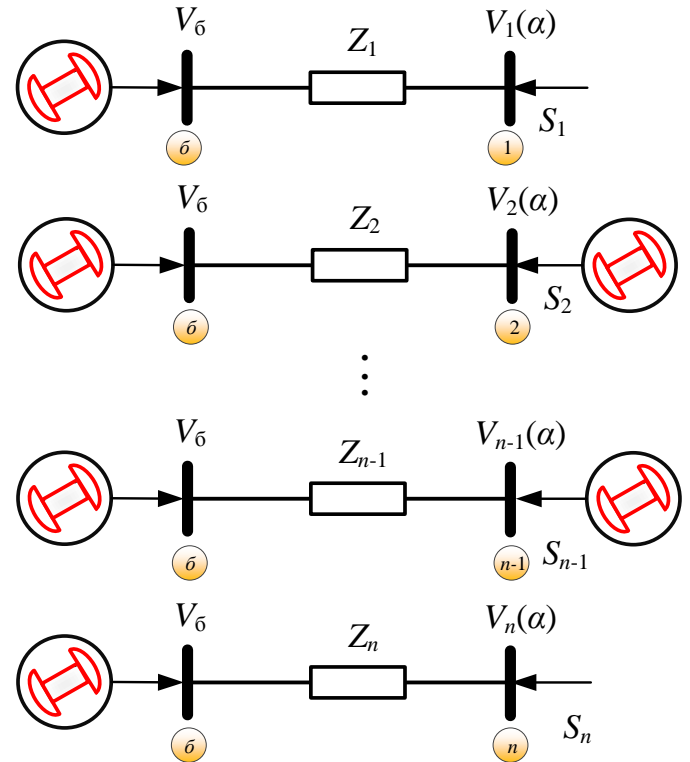

$\sigma / b$

Pис 5. Преобразование многоузловой схемы: а) обобщенная многоузловая схема; б) эквивалентные двухузловые схемы

Fig. 5. Converting a multi-node power system: a) generalized multi-node power system; b) equivalent two-node system

При некоторых допущениях напряжение каждого узла системы можно представить в виде

$$
V_{i}=V_{\sigma}+\frac{Z_{i} S_{i}^{*}}{V_{i}^{*}}
$$

Уравнение (30) можно привести к виду

$$
U_{i}=1+\frac{\sigma_{i}}{U_{i}^{*}},
$$

где $U_{i}=\frac{V_{i}}{V_{\sigma}}$ и $U_{i}^{*}=\frac{V_{i}^{*}}{V_{\sigma}^{*}}-$ напряжения, приведенные к базисному; $\sigma_{i}=\frac{Z_{i} S_{i}^{*}}{\left|V_{\tilde{\sigma}}\right|^{2}}-$ комплексный коэффициент, характеризующий состояние узла в электрической сети, рассчитываются для каждого конкретного узла сети [23].

Коэффициенты $\sigma_{i}$ ( $\sigma$-индексы) могут быть вычислены на основе результатов расчета метода голоморфного погружения. Для этого $\sigma$-индексы можно 
представить в виде функций от параметра погружения $\alpha$ :

$$
\sigma_{i}(\alpha)=\sum_{i=0}^{n} \sigma_{i}[n] \alpha^{n}=\sigma_{i}[0]+\sigma_{i}[1] \alpha+\ldots+\sigma_{i}[n] \alpha^{n} .
$$

Расположение $\sigma_{i}$-индексов относительно параболы $\sigma_{R} \leq \sigma_{I}^{2}-\frac{1}{4}$ позволяет оценить возможность существования системы уравнений многоузловой схемы. Изменение значения параметра погружения позволяет проследить перемещения каждого $\sigma_{i}$-индекса на графике до границы устойчивости, тем самым определить критическое значение коэффициента.

Получим выражение для расчета коэффициентов функции $\sigma(\alpha)$. Для этого осуществим голоморфное погружение параметра $\alpha$ в уравнение (31) следующим образом:

$$
U_{i}(\alpha)=1+\frac{\sigma_{i}(\alpha)}{U_{i}^{*}\left(\alpha^{*}\right)}=1+\sigma_{i}(\alpha) W_{i}^{*}\left(\alpha^{*}\right) .
$$

Заменяя функции комплексных переменных степенными рядами, получаем следующее выражение

$$
\begin{gathered}
\sum_{i=0}^{n} U[n] \alpha^{n}=1+\sum_{i=0}^{n} \sigma[n] \alpha^{n} \sum_{i=0}^{n} W^{*}[n] \alpha^{n}= \\
=1+\sum_{k=0}^{n} \sigma[k] W^{*}[n-k] \alpha^{n} .
\end{gathered}
$$

Раскладывая суммы в (32) и приравнивая коэффициенты при одинаковых степенях $\alpha^{n}$ с левой и правой частей равенства, получаем:

$$
\begin{gathered}
U[0]=1+\sigma[0] W^{*}[0] \\
U[1]=\sigma[0] W^{*}[1]+\sigma[1] W^{*}[0] \\
\vdots \\
U[n]=\sum_{k=0}^{n} \sigma[k] W^{*}[n-k]= \\
=\sum_{k=0}^{n-1} \sigma[k] W^{*}[n-k]+\sigma[n] W^{*}[0] .
\end{gathered}
$$

Из уравнений (33) можно составить общую формулу для вычисления $n$-го коэффициента ряда функции $\sigma(\alpha)$ :

$$
\sigma[n]=\left\{\begin{array}{c}
\frac{U[0]-1}{W^{*}[0]}, n=0 ; \\
\frac{U[n]-\sum_{k=0}^{n-1} \sigma[k] W^{*}[n-k]}{W^{*}[0]}, n \geq 1 .
\end{array}\right.
$$

Для вычисления значения параметра $\sigma$ рассчитываем аппроксимацию Паде $\sigma=\left.[L / M]_{\sigma(\alpha)}\right|_{\alpha=1}$.

Также на графике можно визуально показать, в каких пределах располагаются модули напряжений в узлах. Из выражения (31) следует, что все точки одного и того же модуля напряжения $U^{\prime}$, приведенного к напряжению базисного узла, описывают окружность радиуса $U^{\prime}$, смещенную по действительной оси на $U^{\prime 2}$ и касающуюся граничной параболы:

$$
\sigma=-U^{\prime} e^{-i \delta}+U^{\prime 2} \text { или }\left(\sigma_{R}-U^{\prime 2}\right)^{2}+\sigma_{I}^{2}=U^{\prime 2} \text {. }
$$

Для визуального выявления узлов, напряжение в которых отклоняется от допустимых эксплуатационных значений, на графике можно отобразить окружности, соответствующие допустимым отклонениям. Точки внутри окружности соответствуют узлам с напряжением выше напряжения, для которого построена окружность.

Для рассматриваемой тестовой схемы рассмотрим два случая: нормальный и предельный режим. Выполним расчет для рассматриваемого режима (рис. 3). Из графика на рис. $6, a$ видно, что точки, характеризующие каждый узел, располагаются в пределах параболы. Это говорит о том, что система уравнений совместна и устойчивое решение существует.
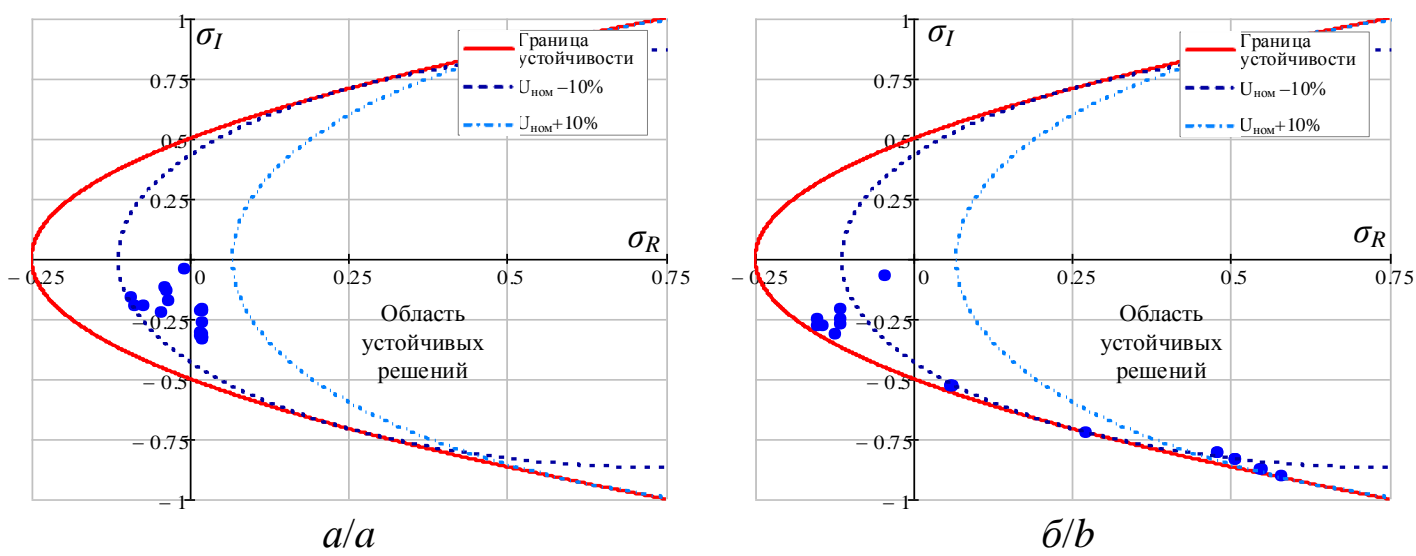

Рис 6. График визуальной оценки возможности существования режима: а) нормальный режим, $S_{\text {н14 }}=2+j 1 \mathrm{MB} \cdot A$; б) предельный режим, $S_{\text {н14 }}=36+j 12 \mathrm{MB} \cdot \mathrm{A}$

Fig. 6. Picture of visual estimation of existence of a steady-state solution: a) normal operation, $S_{n 14}=2+j 1 M V \cdot A$; b) limit load operation, $S_{n 14}=36+j 12 \mathrm{MV} \cdot \mathrm{A}$ 
Предельный режим получаем путем увеличения нагрузки в узле 14 , при этом максимальное значение мощности, при которой режим считается методом Ньютона-Рафсона со стартовым алгоритмом, $S_{\text {н14 }}=36+j 12 \mathrm{MB} \cdot$ А. Из рис. 6 , б видно, что для данного предельного режима точки сдвинулась ближе к параболе, часть точек практически лежит на параболе, что говорит о том, что режим действительно предельный. Следует заметить, что, говоря о многоузловой схеме, нельзя однозначно сказать, что нарушается устойчивость, если хотя бы одна из точек лежит вне параболы. Совокупность двухузловых математических моделей электрической сети неполно описывает действительное состояние реальной многоузловой модели: не учтены статические характеристики нагрузки, действие регуляторов напряжения, изменение положений автоматически регулируемых переключателей ответвлений трансформаторов и т. д. Поэтому, как показали расчеты, в реальной сети режим может существовать в то время, когда условие (29) не выполняется и одна или несколько точек выходят за пределы параболы. Более полный анализ статической устойчивости необходимо выполнять по якобиану, сов- падающему со свободным членом характеристического уравнения при соблюдении определенных условий [3]. Рассмотренный графический способ позволяет произвести примерную оценку возможности существования режима, а также ориентировочно выявить слабые узлы, т. е. узлы, которые наиболее близко расположены к параболе и изменение нагрузки и генерации в которых может привести к нарушению устойчивости.

В качестве дополнительного способа оценки существования системы уравнений можно применить анализ расположения нулей и полюсов разложения Паде на комплексной плоскости [28]. Из рис. 7 видно, что критерием приближения решения к границе устойчивости является приближение корней, расположенных вдоль действительной оси, к окружности радиуса $R=1$ справа. При этом самый левый корень по действительной оси при $\alpha>0$ определяет точку ветвления, которая характеризует предельное значение коэффициента $\alpha$, на которое пропорционально можно увеличить нагрузку и генерацию, чтобы решение существовало.
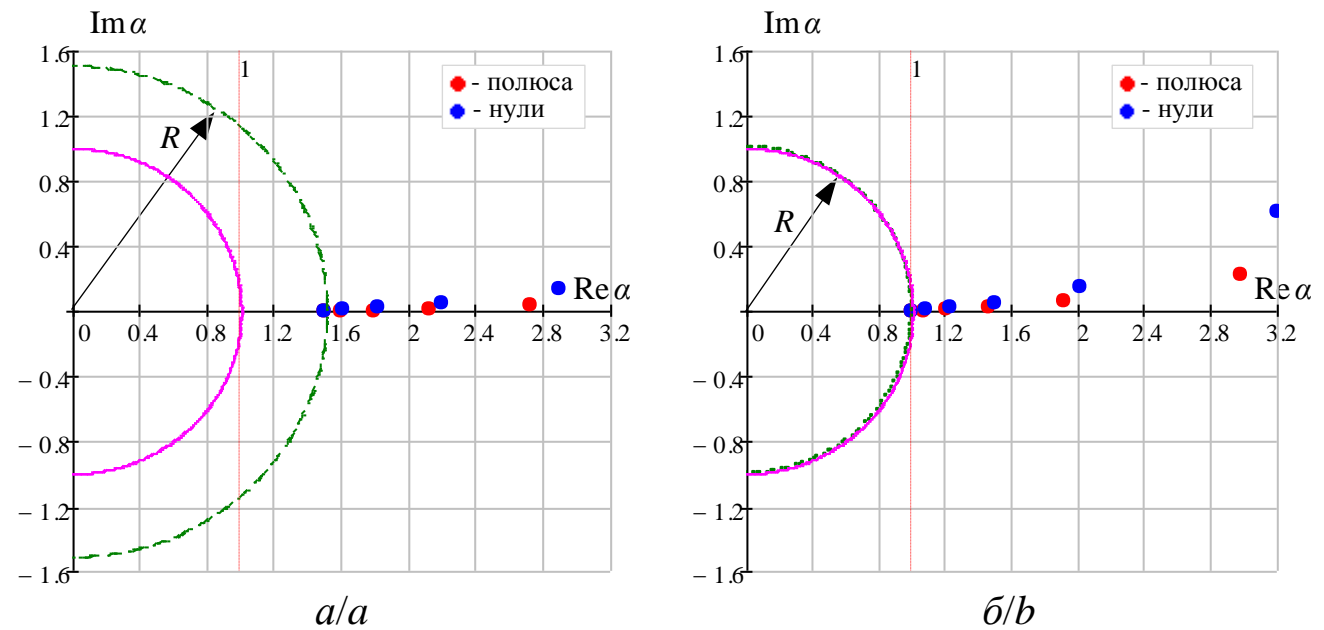

Рис 7. Расположение полюсов и нулей полиномов аппроксимации Паде функции $U_{19}(\alpha):$ a) нормальный режим, $S_{\text {н14 }}=2+j 1 \mathrm{MB} \cdot \mathrm{A}$; б) предельный режим, $S_{\text {н14 }}=36+j 12 \mathrm{MB} \cdot \mathrm{A}$

Fig. 7. Location of poles and zeros of the Padé approximants polynomials of the function $U_{19}(\alpha):$ a) normal operation, $S_{n 14}=2+j 1 M V \cdot A$; b) limit load operation, $S_{n 14}=36+j 12 M V \cdot A$

Значения нулей и полюсов аппроксиманта Паде зависят от вычисленных коэффициентов полиномов числителя и знаменателя аппроксимации Паде. При определённом количестве членов исходного степенного ряда можно получить значения нулей и полюсов, по расположению которых невозможно оценить существование режима из-за их хаотичного расположения на плоскости (рис. 8, a). Применяя регуляризацию при вычислении коэффициентов полинома знаменателя аппроксимации Паде, согласно формуле (28), можно улучшить решение. При этом значения нулей и полюсов будут зависеть от выбранного параметра регуляризации $\lambda$ (рис. $8, \sigma-2$ ). Необходимо подобрать параметр регуляризации $\lambda$ так, чтобы корни расположились по действительной. Оптимальное зна- чение параметра регуляризации $\lambda=10^{-15}$, при $\lambda=10^{-25}$ корни отдаляются от действительной оси.

Точка ветвления без расчета полюсов и нулей аппроксимации Паде может быть приближенно определена по критерию Фабри [28]

$$
R_{i}=\lim _{n \rightarrow \infty} \frac{\left|c_{n}\right|}{\left|c_{n+1}\right|},
$$

где $c_{0}, c_{1}, \ldots, c_{n}-$ коэффициенты функции напряжения $i$-го узла $U_{i}(\alpha)=c_{0}+c_{1} \alpha+\ldots+c_{n} \alpha^{n}$.

Точки ветвления рассчитываются для каждого узла схемы, при этом в качестве предельного значения выбирается самое минимальное значение $R_{i}$.

Стоит отметить, что величину $\max \left(1 / R_{i}\right)$ можно рассматривать как коэффициент напряженности ре- 
жима $k_{\text {н }}$ т. е. показатель запаса статической устойчивости энергосистемы, численно характеризующий напряженность режима. При $k_{\mathrm{H}}<1$ режим существует, при $k_{\mathrm{H}}=1$ - режим предельный, при $k_{\mathrm{H}}>1$ - режим не существует. В таблице представлены результаты расчета для рассматриваемой схемы.

\section{Заключение}

В работе представлен аналитический метод расчета установившихся режимов - метод голоморфного погружения. Рассмотренный метод существенно повышает эффективность решения задачи расчета установившихся режимов распределительных сетей нефтегазовых месторождений. Преимуществом метода является то, что для него не нужно задавать начальное приближение, как в классических итерационных методах.

На примере тестовой схемы показано, что погрешность меньше в случае, если неизвестные параметры описываются сходящимися рядами. За счет использования аппроксимации Паде можно получить корректный результат с меньшей погрешностью. Аппроксимация Паде обеспечивает хорошее приближение вне радиуса сходимости выражения степенного ряда, а также ускоряет сходимость.
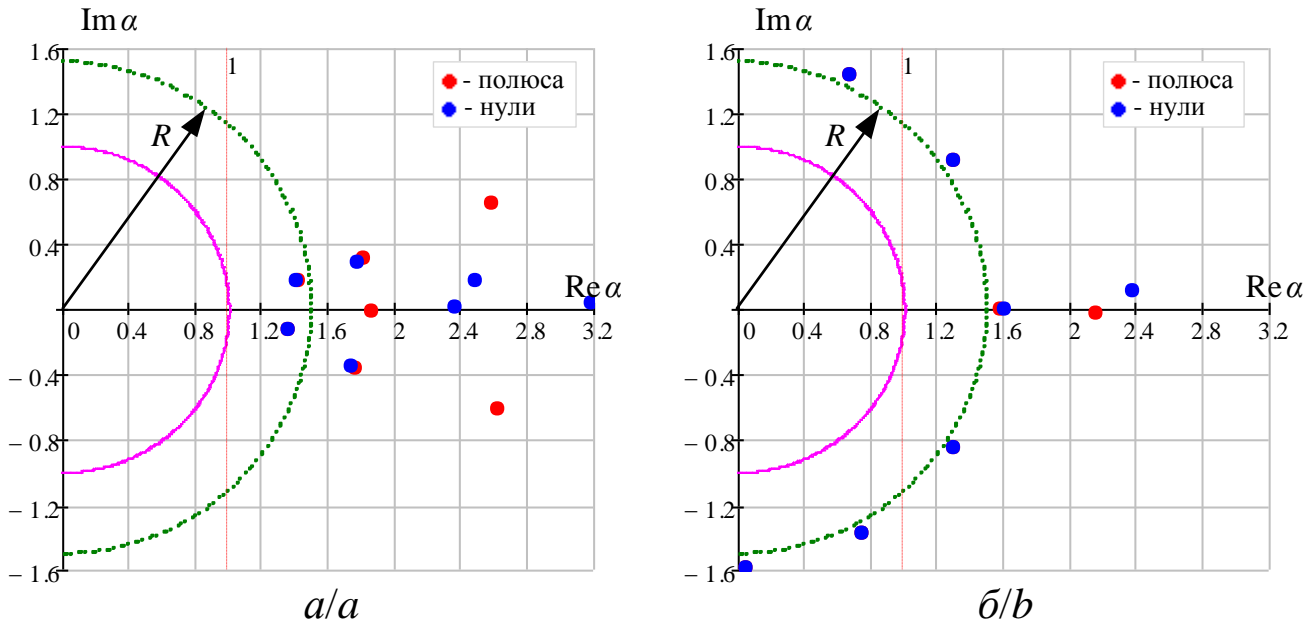

$\operatorname{Im} \alpha$

$\operatorname{Im} \alpha$
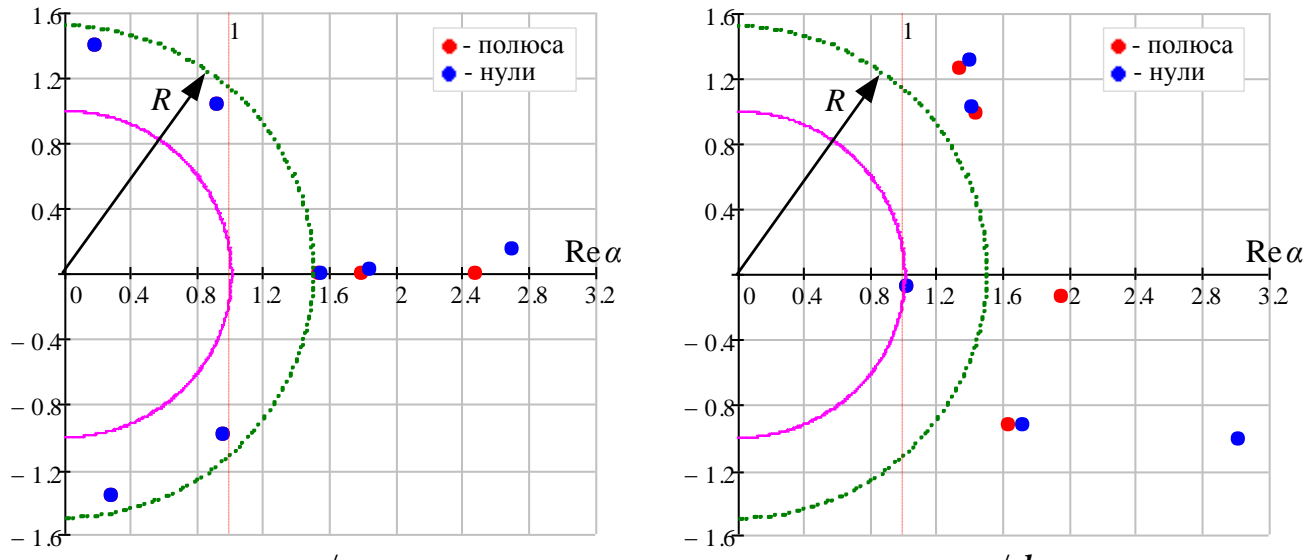

2/d

Pис. 8. Расположение полюсов и нулей полиномов аппроксимации Паде функиии $U_{19}(\alpha)$ в нормальном режиме при $n=32:$ а) без регуляризацุии; б) с регуляризацчией, $\lambda=10^{-10}$; в) с регуляризацчией, $\lambda=10^{-15}$; г) с регуляризацчией, $\lambda=10^{-25}$

Fig. 8. Location of poles and zeros of the Padé approximants polynomials of the function $U_{19}(\alpha)$ : a) without regularization; b) with regularization, $\left.\lambda=10^{-10} ; c\right)$ with regularization, $\left.\lambda=10^{-15} ; d\right)$ with regularization, $\lambda=10^{-25}$

Таблица. Оценивание существования режимов по полюсам и нулям полиномов аппроксимаций Паде

Table. $\quad$ Estimation of power system stability using poles and zeros of Padé approximants polynomials

\begin{tabular}{|c|c|c|c|c|}
\hline Режим/State & Мин. полюс/Min. pole & Мин. ноль/Min. zero & $\min \left(R_{i}\right)$ & $\max \left(1 / R_{i}\right)$ \\
\hline Нормальный режим, & & & & \\
$S_{\text {н14 }}=2+j 1 M B \cdot A$ \\
$\begin{array}{c}\text { Normal operation, } S_{n 14}=2+j 1 \\
M V \cdot A\end{array}$ & $1,5086-j 9,981716 \cdot 10^{-4}$ & $1,512911+j 2,587827 \cdot 10^{-4}$ & 1,509797 & 0,662341 \\
\hline $\begin{array}{c}\text { Предельный режим, } \\
S_{\text {н14 }}=36+j 12 M B \cdot A \\
\text { Limit load operation, } \\
S_{n 14}=36+j 12 M V \cdot A\end{array}$ & $1,005364-j 3,261143 \cdot 10^{-3}$ & $1,00707+j 1,985935 \cdot 10^{-3}$ & 1,003287 & 0,996724 \\
\hline
\end{tabular}




\begin{tabular}{|c|c|c|c|c|}
\hline $\begin{array}{c}\text { Нефизическое решение, } \\
S_{\text {н14 }}=37+j 13 M B \cdot A \\
\text { Non-physical solutions, } \\
S_{n 14}=37+j 13 M V \cdot A\end{array}$ & $0,98805-j 3,242884 \cdot 10^{-3}$ & $0,989631+j 1,953492 \cdot 10^{-3}$ & 0,985943 & 1,014257 \\
\hline
\end{tabular}

Метод открывает новые возможности анализа состояний энергосистем, решения проблемы существования, множественности решения и анализа устойчивости за счет использования концепций применения алгебраических кривых и комплексного анализа.

\section{СПИСОК ЛИТЕРАТУРЫ}

1. Вычислительные модели потокораспределения в электрических системах / Б.И. Аюев, В.В. Давыдов, П.М. Ерохин, В.Г. Неуймин. - М.: Изд-во «Флинта», 2008. - 256 с.

2. Тарасов В.И. Теоретические основы анализа установившихся режимов электроэнергетических систем. - Новосибирск: Наука, 2002. - 344 c.

3. Идельчик В.И. Расчеты установившихся режимов электрических систем. - М.: Энергия, 1977. - 192 с.

4. Архипова О.В., Ковалев В.З., Хамитов Р.Н. Методика моделирования регионально обособленного электротехнического комплекса // Известия Томского политехнического университета. Инжиниринг георесурсов. - 2019. - Т. 330. - № 1. C. $173-180$.

5. Гуревич Ю.Е., Либова Л.Е., Окин А.А. Расчеты устойчивости и противоаварийной автоматики в энергосистемах. - М.: Энергоатомиздат, 1990. - 390 c.

6. Новые технологии и современное оборудование в энергетике нефтегазовой промышленности / И.В. Белоусенко, Г.Р. Шварц, С.Н. Великий, М.С. Ершов, А.Д. Яризов. - М.: ООО «НедраБизнесцентра», 2007. - $478 \mathrm{c}$.

7. Тарасов В.И. Методы минимизации ньютоновского типа для расчета установившихся режимов электроэнергетических систем. - Новосибирск: Наука, 2001. - 168 с.

8. Trias A. The holomorphic embedding load flow method // Power and Energy Society General Meeting. - San Diego, July 2012. P. 1-8.

9. Trias A. Fundamentals of the holomorphic embedding load-flow method. URL: https://arxiv.org/abs/1509.02421 (дата обращения: 01.09.2020).

10. Исаев Ю.Н., Кабалин Д.А. Голоморфное погружение как аналитический метод расчета электрических сетей нефтяных и газовых месторождений // Известия Томского политехнического университета. Инжиниринг георесурсов. - 2020. T. 331. - № 4. - C. 115-125.

11. Шабат Б.В. Введение в комплексный анализ. Ч. 1: Функции одного переменного. - М.: Наука, 2004. - 336 c.

12. Subramanian M.K., Feng Y., Tylavsky D. PV bus modeling in a holomorphically embedded power-flow formulation // 2013 North American Power Symposium (NAPS). - Manhattan, 2013. - P. 1-6.

13. The holomorphic embedding method applied to the power-flow problem / S. Rao, Y. Feng, D.J. Tylavsky, M.K. Subramanian // IEEE Transactions on Power Systems. 2016. - V. 31. - № 5. P. 3816-3828.

14. Ali Reza Abbasi. Probabilistic load flow based on holomorphic embedding, kernel density estimator and saddle point
Предложенные уравнения голоморфного погружения не учитывают статических характеристик нагрузки. В дальнейшем планируется расширить модель с учетом статических характеристик нагрузки.

approximation including correlated uncertainty variables // Electric Power Systems Research. - 2020. - V. 183. - P. 106178.

15. Santos A.C., Freitas F.D., Fernandes L.F.J. Holomorphic embedding approach as an alternative method for solving the power flow problem // 2017 Workshop on Communication Networks and Power Systems (WCNPS). - Brasilia, 2017. - P. 1-4.

16. Remote voltage control using the holomorphic embedding load flow method / C. Liu, N. Qin, K. Sun, C.L. Bak // IEEE Transactions on Smart Grid. - 2019. - V. 10. - № 6. - P. 6308 6319.

17. Бейкер Дж. мл., Грейвс-Моррис П. Аппроксимация Паде. - М.: Мир, 1986. -502 c.

18. Суетин С.П. Аппроксимации Паде и эффективное аналитическое продолжение степенного ряда // УМН. - 2002. - Т. 57. Вып. 1 (343). - С. 45-142.

19. Никишин Е.М., Сорокин В.Н. Рациональные аппроксимации и ортогональность. - М.: Наука, 1988. -258 с

20. Stahl H. Orthogonal polynomials with complex-valued function. I, II // Constr. Approx. - 1986. - V. 2. - P. 225-251.

21. Stahl H. Domains associated with an analytic function. I // Complex Variables Theory Appl. - 1985. - V. 4 (4). - P. 311-324.

22. Тихонов А.Н., Арсенин В.Я. Методы решения некорректно поставленных задач. - М.: Наука, 1986. - 286 с.

23. Sigma algebraic approximants as a diagnostic tool in power networks: US Patent, № 9563722 B2, 2017.

24. Rao S.D., Tylavsky D.J., Feng Y. Estimating the saddle-node bifurcation point of static power systems using the holomorphic embedding method // International Journal of Electrical Power \& Energy Systems. - 2017. - V. 84. - P. 1-12.

25. Rao S., Tylavsky D., et al. Two-bus holomorphic embedding method based equivalents and weak-bus determination. 2017. URL: https://arxiv.org/abs/1706.01298 (дата обращения: 01.09.2020)

26. Lai Q., Liu C., Sun K. A network decoupling method for voltage stability analysis based on holomorphic embedding. URL: https://arxiv.org/abs/2003.12287 (дата обращения: 01.09.2020).

27. Online voltage stability assessment for load areas based on the holomorphic embedding method / Chengxi Liu, Bin Wang, Fengkai Hu, Kai Sun, Claus Leth Bak // IEEE Transactions on Power Systems. - 2018. - V. 33. - № 4. - P. 3720-3734.

28. Suetin S.P., Baghsorkhi S.S. Embedding AC power flow in the complex plane. Part I: Modelling and mathematical foundation. 2016. - 13 p. URL: https://arxiv.org/abs/1604.03425 (дата обращения: 01.09.2020)

Поступила 05.02.2021 г.

\section{Информация об авторах}

Исаев Ю.Н., доктор физико-математических наук, профессор отделения электроэнергетики и электротехники Инженерной школы энергетики Национального исследовательского Томского политехнического университета.

Кабалин Д.А., аспирант отделения электроэнергетики, Инженерной школы энергетики Национального исследовательского Томского политехнического университета.

Филипас $\boldsymbol{A} . \boldsymbol{A}$., кандидат технических наук, доцент отделения автоматизации и робототехники Инженерной школы информационных технологий и робототехники Национального исследовательского Томского политехнического университета. 
UDC 621.314.58:681.5.015

\title{
HOLOMORPHIC EMBEDDING AS ANALYTICAL TECHNIQUE FOR CALCULATING ELECTRIC GRIDS OF OIL AND GAS DEPOSITS AND ASSESSING THEIR STABILITY
}

\author{
Yusup N. Isaev 1 , \\ isaev_yusup@mail.ru
}

Dmitry A. Kabalin'1, mitya.kabalin@gmail.com

\author{
Alexander A. Filipas', \\ filipas@tpu.ru
1 National Research Tomsk Polytechnic University, 30, Lenin avenue, Tomsk, 634050, Russia.

To effectively solve the problems of operational dispatch control of the operating modes of the unified power system, its individual power systems and power districts, in particular, oil production power districts, it is required to carry out calculations of the established modes of electrical networks. In addition, along with the calculations of steady-state regimes, it is important to study the stability of the power grid operation. The convergence and rate of convergence of widely used iterative methods for calculating steady-state modes depend on many operating and design factors determined by the network and mode parameters, the choice of initial approximations, and the method for specifying the initial data. Therefore, the development of new methods that make it possible to calculate all steady-state regimes are of significant practical interest. One of the promising techniques is the holomorphic imbedding method. In this method, the unknown parameters of the nodes are represented in the form of holomorphic functions, which can be written in the form of power series, the coefficients of which are calculated using recurrent expressions, and the problem is reduced to finding the coefficients of the power series. In a previously published article by the authors, the method is considered for a circuit with load nodes. For a complete correct analysis of the modes of real power systems, it is necessary to show how to calculate for generating units. The paper presents recurrent expressions for calculating the unknown coefficients of the holomorphic functions of the unknown parameters of the system of steady-state equations for the load and generator nodes. The expressions obtained, in contrast to those proposed in the works of other authors, are more general. The principle of forming a matrix equation for finding unknown coefficients with the division of complex parameters into real and imaginary parts is shown. A method for obtaining converging power-law in some cases is proposed. On the example of a test power system, the advantage over the Newton-Raphson method is shown. We consider the question of evaluating the existence of a solution to the system of equations of the steady state for a multinode network based on a sigma-graph. An approach is proposed to determine the indicator of the static stability margin of the power system, based on the Fabry criterion.

The aim of the research is to apply the analytical method of holomorphic imbedding to calculate an electrical circuit containing load and generator units; to evaluate the influence of the number of calculated power series coefficients on the accuracy of the obtained solution, and also to consider ways to increase the numerical accuracy of the solution, consider the question of evaluating the existence of a solution to the system of equations of the steady state for a multi-node network based on the analysis of power series.

Methods: Taylor expansion, analytic continuation, Padé approximation, solving algebraic equations by the recursive method.

Results. Using the example of a scheme with a poorly conditioned Jacobi matrix, in which the Newton-Raphson method does not converge from a flat start, the advantage of the holomorphic immersion method is shown. The influence of the number of members of the power series on the calculation error is shown. For the considered scheme, a graphical estimate of the existence of a solution to the system of equations is performed.

Conclusions. For load and generator nodes, the unknown parameters can be represented as holomorphic functions, which can be written down as a Taylor series, the coefficients of which are calculated using recurrent expressions. Partial consideration of shunts to the ground in the diagonal elements of the matrix of successive conductances makes it possible to obtain converging power series in some cases. The considered graphical method for assessing the possibility of the regime existence allows us to make a rough estimate. Unlike classical iterative methods, an initial approximation is not specified for the holomorphic immersion method.

\section{Key words:}

Power system, steady-state process, stability, nonlinear equations, holomorphic immersion, Padé expansion, convergence.

\section{REFERENCES}

1. Ayuev B.I., Davydov V.V., Erokhin P.M., Neuiymin V.G. Vychislitelnye modeli potokoraspredeleniya $v$ elektricheskikh sistemakh [Power flow computing models in power systems]. Moscow, Nauka Publ., 2008. 256 p.

2. Tarasov V.I. Teoreticheskie osnovy analiza ustanovivshikhsya rezhimov elektroenergeticheskikh sistem [Theoretical bases of analysis of steady-state modes of electric power systems]. Novosibirsk, Nauka Publ., 2002. 344 p.

3. Idelchik V.I. Raschety ustanovivshikhsya rezhimov elektricheskikh sistem [Calculations of steady-state modes of electric systems] Moscow, Energia Publ., 1977. 192 p.

4. Arkhipova O.V., KovalevV.Z., Khamitov R.N. Methodology of modeling regionally isolated electrotechnical complex. Bulletin of the Tomsk Polytechnic University. Geo Assets Engineering, 2019, vol. 330, no. 1, pp. 173-180. In Rus.

5. Gurevich Yu.E., Libova L.E., Okin A.A. Raschety ustoychivosti $i$ protivoavariynoy avtomatiki $v$ energosistemakh [Calculation of stability and emergency automation in power systems]. Moscow, Energoatomizdat Publ., 1990. 390 p.

6. Belousenko I.V., Schwartz G.R., Great S.N., Ershov M.S. Yarizov A.D. Novye tekhnologii i sovremennoe oborudovanie v energetike neftegazovoy promyshlennosti [New technologies and modern equipment in the energy sector of the oil and gas industry]. Moscow, Nedra-Biznestsentra Publ., 2007. 478 p.

7. Tarasov V.I. Metody minimizatsii nyutonovskogo tipa dlya rascheta ustanovivshihsya rezhimov elektroenergeticheskih sistem [Newton type minimization methods for calculation steady states of electric power systems]. Novosibirsk, Nauka Publ., 2001. 168 p. 
8. Trias A. The holomorphic embedding load flow method. Power and Energy Society General Meeting. San Diego, July 2012. pp. $1-8$.

9. Shabat B.V. Vvedenie v kompleksny analiz. Ch. 1: Funktsii odnogo peremennogo [Introduction to complex analysis. P. 1: Functions of one variable]. Moscow, Nauka Publ., 2004. 336 p.

10. Trias A. Fundamentals of the holomorphic embedding load-flow method. Available at: https://arxiv.org/abs/1509.02421 (accessed 1 September 2020).

11. Isaev Yu.N., Kabalin D.A. Holomorphic embedding as analytical technique for calculating electric grids of oil and gas deposits. Bulletin of the Tomsk Polytechnic University. Geo Assets Engineering 2020, vol. 331, no. 4, pp. 115-125. In Rus.

12. Subramanian M.K., Feng Y., Tylavsky D. PV bus modeling in a holomorphically embedded power-flow formulation. 2013 North American Power Symposium (NAPS). Manhattan, 2013. pp. 1-6.

13. Rao S., Feng Y., Tylavsky D.J., Subramanian M.K. The holomorphic embedding method applied to the power-flow problem. IEEE Transactions on Power Systems, 2016, vol. 31, no. 5, pp. 3816-3828

14. Ali Reza Abbasi. Probabilistic load flow based on holomorphic embedding, kernel density estimator and saddle point approximation including correlated uncertainty variables. Electric Power Systems Research, 2020, vol. 183, pp. 106178.

15. Santos A.C., Freitas F.D., Fernandes L.F.J. Holomorphic embedding approach as an alternative method for solving the power flow problem. 2017 Workshop on Communication Networks and Power Systems (WCNPS). Brasilia, 2017. pp. 1-4.

16. Liu C., Qin N., Sun K., Bak C.L. Remote voltage control using the holomorphic embedding load flow method. IEEE Transactions on Smart Grid, 2019, vol. 10, no. 6, pp. 6308-6319.

17. Baker G.A., Graves-Morris P. Approksimatsia Pade [Padé approximants]. Moscow, Mir Publ., 1986. 502 p.

18. Suetin S.P. Padé approximants and the effective analytic continuation of a power series. Russian Math. Surveys, 2002, vol. 57, no. 1, pp. 45-142.

Information about the authors

Yusup N. Isaev, Dr. Sc., professor, National Research Tomsk Polytechnic University.

Dmitry A. Kabalin, postgraduate student, National Research Tomsk Polytechnic University.

Alexander A. Filipas, Cand. Sc., associate professor, National Research Tomsk Polytechnic University.
19. Nikishin E.M., Sorokin V.N. Ratsionalnye approksimatsii $i$ ortogonalnost [Rational approximations and orthogonality]. Moscow, Nauka Publ., 1988. 258 p.

20. Stahl H. Orthogonal polynomials with complex-valued function. I, II. Constr. Approx., 1986, vol. 2, pp. 225-251.

21. Stahl H. Domains associated with an analytic function. I. Complex Variables Theory Appl., 1985, vol. 4 (4), pp. 311-324.

22. Tihonov A.N., Arsenin V.Ya. Metody resheniya nekorrektno postavlennyh zadach [Methods of solution of ill-posed problems]. Moscow, Nauka Publ., 1986. 286 p.

23. Trias A. Sigma algebraic approximants as a diagnostic tool in power networks. US Patent, no. 9563722 B2, 2017.

24. Rao S.D., Tylavsky D.J., Feng Y. Estimating the saddle-node bifurcation point of static power systems using the holomorphic embedding method. International Journal of Electrical Power \& Energy Systems, 2017, vol. 84, pp. 1-12.

25. Rao S., Tylavsky D. Two-bus holomorphic embedding method based equivalents and weak-bus determination. 2017. Available at: https://arxiv.org/abs/1706.01298 (accessed 1 September 2020).

26. Lai Q., Liu C., Sun K. A network decoupling method for voltage stability analysis based on holomorphic embedding. 2020. Available at: https://arxiv.org/abs/2003.12287 (accessed 1 September 2020)

27. Chengxi Liu, Bin Wang, Fengkai Hu, Kai Sun, Claus Leth Bak. Online voltage stability assessment for load areas based on the holomorphic embedding method. IEEE Transactions on Power Systems, 2018, vol. 33, no. 4, pp. 3720-3734.

28. Suetin S.P., Baghsorkhi S.S. Embedding AC power flow in the complex plane. P. I: Modelling and mathematical foundation. 2016. 13 p. Available at: https://arxiv.org/abs/1604.03425 (accessed 1 September 2020).

Received: 5 February 2021. 